\title{
The Padé-Laplace formalism for carbon and climate responses
}

\author{
I. G. Enting ${ }^{a}$ \\ ${ }^{\text {a } C S I R O ~ C l i m a t e}$ Science Centre, Oceans and Atmosphere, Aspendale, VIC, Australia \\ Email: ian.g.enting@gmail.com
}

\begin{abstract}
Impulse response functions have been used extensively in studies of $\mathrm{CO}_{2}$ (carbon dioxide), the carbon cycle and its interactions with the climate system. The response functions have been presented as compact descriptions of model behaviour as well as being used computationally, including as representations of subsystems in models.
\end{abstract}

Response functions for emissions of greenhouse gases have also been important because of being used to define the Global Warming Potential which is used to compare the importance of different greenhouse gases.

The Laplace transform formalism is convenient for analysing aspects of response functions because convolutions transform into products of transforms. The Laplace transform has been used in various ways to illustrate connections within the carbon-climate system, but generally has not been used for quantitative calculations.

This paper explores the use of Laplace transforms as a computational tool for investigating $\mathrm{CO}_{2}$ and climate. The Padé-Laplace approach consist of taking a Maclaurin series or Taylor series expansion of the Laplace transform of a response function and fitting the leading terms to a ratio of polynomials. Such approximations are known as Padé approximants.

Approximants of appropriate order can be expressed as sums of partial fractions. Therefore such approximations can be used to give low-order expressions as sums of exponentials in the time domain.

Results presented here illustrate a number of important cases:

(i) the impulse response for $\mathrm{CO}_{2}$ is transformed to give an expression for what is called the concentration feedback - the extent to which increases in atmospheric $\mathrm{CO}_{2}$ cause uptake of carbon into land and ocean systems. The low-order approximants give a better representation than the common practice of expressing this feedback as a constant known as the beta-factor.

(ii) The feedback around the loop of the coupled carbon-climate system is expressed as a gain operator whose response is derived by combining model estimates of $\mathrm{CO}_{2}$ response with and without feedback. Low-order approximants provide a way of estimating the gain, when combining ice-core $\mathrm{CO}_{2}$ data with paleo-temperature data to estimate the climate-to-carbon influence - a quantity that is commonly approximated as a constant termed gamma.

Most calculations presented here use Padé approximants to Maclaurin series of response functions in the transform domain. Consequently, they most accurately capture the long-term behaviour in the time domain relevant for stabilising climate forcing. Applying the Padé-Laplace method using Taylor series expansions gives the potential for investigations of decadal to century scale global change. Such studies are in progress.

Keywords: Carbon dioxide, climate, Laplace transforms 
I. G. Enting, Pade-Laplace in carbon-climate interactions.

\section{INTRODUCTION}

Representing dynamical systems using impulse response functions gives the maximum degree of generality for causal linear systems. Such representations have been used in studies of carbon dioxide and climate, both as descriptions of behaviour and as components of numerical models (e.g. Meinshausen et al. 2011). Impulse response functions that relate emissions to concentrations also underlie metrics such as the global warming potentials that characterise the relative importance of emissions of different greenhouse gases. An extensive intercomparison of the response function for $\mathrm{CO}_{2}$ was reported by Joos et al. (2013).

The Laplace transform formalism is particularly useful in representing impulse response relations because convolution integrals transform into products of transforms. A number of studies have used the Laplace transform to define structural relations between different aspects of the carbon cycle (Enting \& Mansbridge 1987. Enting 1990, Enting et al. 1994) and its relation to climate (Enting 2010). However, these studies have explored qualitative relations and have not involved quantitative calculations in the transform domain.

The Padé-Laplace analysis is based on expressing the Laplace transform as a Maclaurin series or Taylor series in the transform variable, and then fitting a Padé approximant (i.e. a ratio of two polynomials) to that series. This can be done with different orders of approximation and provides a way of exploring simpler approximations to complicated expressions for combinations of transforms. The Padé-Laplace technique derives from the study by Yeramian \& Claverie (1987) and its extension by Claverie et al. (1989). The technique has been used in the analysis of integrated circuits (e.g. Feldman \& Freund 1995). Enting \& Clisby (2021) used low-order representations in the transform domain to compare metrics for comparing greenhouse gases.

Section 2 below summarises the Padé-Laplace formalism. The following sections apply this quantitative analysis to specific cases that have been considered in previous qualitative analyses: $\mathrm{CO}_{2}$ responses and the analysis of $\mathrm{CO}_{2}$ response as a concentration feedback in Section 3, deconvolution calculations in Section 4, and the climate-carbon feedback loop in Section 5. Section 6 reviews the estimation of the climate-to-carbon link in the feedback loop. The concluding section notes some of the directions for on-going research after which a table of notation is given.

\section{THE PADE LAPLACE FORMALISM}

A generic response function relation can be written as

$$
Q(t)=M(t)-M(0)=\int_{0}^{t} R\left(t-t^{\prime}\right) S\left(t^{\prime}\right) d t^{\prime}
$$

When applied to the carbon cycle, $M(t)$ represents the $\mathrm{CO}_{2}$ content of the atmosphere, typically in units of $\mathrm{Gt}$ $\mathrm{C}$ (i.e. Pg C), $S(t)$ is the emission rate (e.g. in $\mathrm{Gt} \mathrm{C} /$ year) and $R(t)$ gives the proportion of an initial emission remaining in the atmosphere after time $t$. The time origin of the integration represents a notional pre-industrial equilibrium. Various modifications of this generic form are used in the examples below.

Using a tilde to denote Laplace transforms, equation (1) transforms to

$$
\tilde{Q}(p)=\tilde{R}(p) \tilde{S}(p)
$$

Response functions such as $R(t)$ are often approximated as sums of decaying exponentials. Thus $\tilde{R}(p)$ becomes a sum of terms $b_{j} /\left(p+c_{j}\right)$. Although this is sometimes misunderstood, the decay rates $c_{j}$ will generally not have any physical significance. This is to be expected since the process of fitting sums of exponentials is often ill-conditioned, as illustrated in the classical example from (Lanczos 1956, Ch. 4, Sec. 23) where a sum of 3 exponentials, $f_{3}(t)=0.0951 e^{-t}+0.8607 e^{-3 t}+1.5576 e^{-5 t}$ is closely fitted by a sum of two exponentials, $f_{2}(t)=2.202 e^{-4.45 t}+0.305 e^{-1.58 t}$. The functions are so similar that the difference only becomes apparent when the difference is plotted on an expanded scale as in the solid line in Figure 1.

The Padé-Laplace technique provides a systematic way of providing approximations that may:

(i) avoid the redundancy of high-order representations; and

(ii) lead to simple closed form expressions in the time domain.

The procedure constructs approximations in the transform domain, by making the approximation fit the full expression by matching the leading coefficients of the Maclaurin series in $p$. The particular approximations are ratios of low-order polynomials. When constructed in this way, such approximations are termed Padé approximants. The approximants are characterised by the degrees $N, M$ of the numerator and denominator where the $[M, N]$ approximant fits the first $M+N+1$ terms in the series and the constant term in the 
denominator is normalised to 1 . The $[M, M+1]$ approximants can be expressed as sums of partial fractions, giving a sum of exponentials in the time domain.

Using Padé approximants to construct estimates as a sum of exponentials in this way can include cases (e.g. Enting 1990) where the Maclaurin series did not originally come from a sum of exponentials.

Cases that go beyond scope of this paper can be defined by constructing lower-order approximations that agree in the leading terms of a Taylor series about some chosen $p_{0}$. This form was proposed by Yeramian \& Claverie (1987) where the Taylor series terms were obtained by numerical integration of observational data. For example, the $[1,2]$ approximant fitted to the Taylor series for $\tilde{f}_{3}(p)$ at $p_{0}=1$ approximates $f_{3}(t)$ as $f^{[1,2]}=2.2182 e^{-t / 4.4754}+0.2943 e^{-t / 1.5796}$. The difference from $f_{3}(t)$ is shown as the dotted line in Figure 1 .

\section{Difference in Lanczos cases}

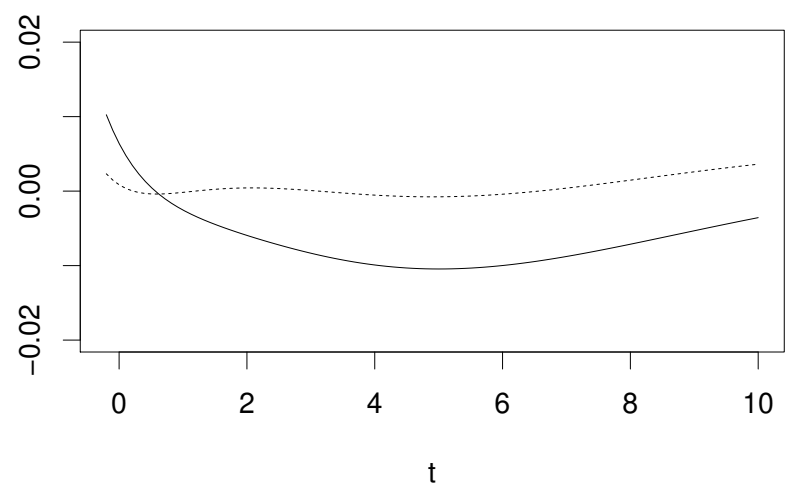

Figure 1. Solid line is difference between the two sums, $f_{3}(t)$ and $f_{2}(t)$ compared by (Lanczos 1956, Ch. 4, Sec. 23). Dashed line is the difference between $f_{3}(t)$ and the sum of two exponentials derived from the $[1,2]$ approximant to $\tilde{f}_{3}(p)$ fitted around $p_{0}=1$.

\section{CARBON DIOXIDE RESPONSE AS CONCENTRATION FEEDBACK}

Oeschger et al. (1980) studied the response of linear carbon cycle models to $\mathrm{CO}_{2}$ emissions that grew exponentially with growth rate $\alpha$. They characterised the partitioning of carbon between atmosphere, land reservoirs and ocean reservoirs as being in the ratio $\left(1: \beta_{\mathrm{L}, \alpha}: \beta_{\mathrm{O}, \alpha}\right)$. For exponentially growing emissions, the cumulative and instantaneous partition ratios are equal. With $Q_{\alpha}, \beta_{\mathrm{L}, \alpha}, \beta_{\mathrm{O}, \alpha}, S_{\alpha}$ as amplitudes of a common $\exp (\alpha t)$ growth, this corresponds to

$$
\tilde{Q}_{\alpha}=\frac{S_{\alpha} / \alpha}{1+\beta_{\mathrm{L}, \alpha}+\beta_{\mathrm{O}, \alpha}}
$$

This relation can be generalised to a Laplace transform relation

$$
\tilde{Q}(p)=\frac{\tilde{S}(0) / p}{1+\tilde{B}_{\mathrm{L}}(p)+\tilde{B}_{\mathrm{O}}(p)}=\tilde{S}(0) / p-\left[\tilde{B}_{\mathrm{L}}(p)+\tilde{B}_{\mathrm{O}}(p)\right] \tilde{Q}(p)
$$

for which the Oeschger et al. result becomes a special case.

In these terms, $\tilde{B}_{\mathrm{L}}(p)$ and $\tilde{B}_{\mathrm{O}}(p)$ describe a negative feedback on the increase of atmospheric $\mathrm{CO}_{2}$, proportional to the $\mathrm{CO}_{2}$ increase, $Q(t)$. The multiplication by $\tilde{B}_{\mathrm{L}}(p)$ and $\tilde{B}_{\mathrm{O}}(p)$ represents convolutions with responses characterising concentration feedback. The $\beta$ factors are sometimes treated as constants (e.g. equation (14) below from Friedlingstein et al. 2003).

The connection between this concentration feedback and the $\mathrm{CO}_{2}$ response functions seems to be little appreciated. For example, Gregory et al. (2009) state that "The concentration to carbon feedback is negative; it has generally received less attention in the literature" [i.e. less than the temperature to carbon feedback]. However, comparison of equations (2) and (4) shows that

$$
p \tilde{R}(p)=\frac{1}{1+\tilde{B}_{\mathrm{L}}(p)+\tilde{B}_{\mathrm{O}}(p)}
$$


which relates the concentration feedback to the widely studied response function via their respective expressions for the airborne fraction.

The feedbacks can be combined in various ways to give a range of different response functions. For example, the introduction of response functions in carbon cycle studies by Oeschger \& Heimann (1983) used oceanonly response, $\tilde{R}_{\mathrm{O}}(p)=1 /\left(1+\tilde{B}_{\mathrm{O}}(p)\right)$. Expressions for combining various different types of carbon cycle response functions were given by Enting et al. (1994) and Enting (2007).

Relation (5) gives

$$
\tilde{B}(p)=\tilde{B}_{\mathrm{L}}(p)+\tilde{B}_{\mathrm{O}}(p)=-1+1 /(p \tilde{R}(p))
$$

and representations of the feedback operator can be obtained from approximants to $\tilde{B}(p)$. Figure 2 shows $\tilde{B}(p)$ calculated using the response $R_{\text {linit }}(t)$ from Enting et al. (1994) and several low-order approximants to the expansion of $\tilde{B}(p)$. Using Maclaurin series means that long-timescale responses dominate the approximants.

\section{Concentration feedback}

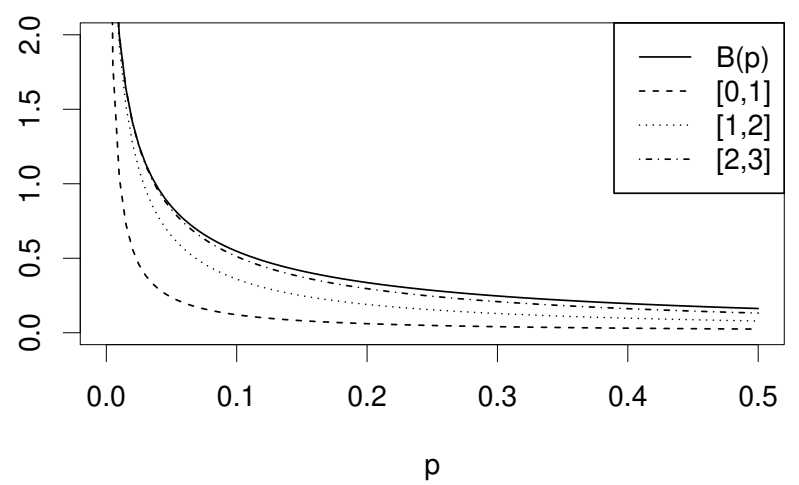

Figure 2. Solid line: $\tilde{B}(p)$ as specified by equation (6) using the response $R_{\text {init }}$ from Enting et al. (1994). Other lines: low-order ([0.1], [1,2], [2,3]) approximants as indicated.

\section{DECONVOLUTION}

In carbon cycle modelling, deconvolution refers to inverting the convolution relation (1) to deduce $S(t)$ given a knowledge of $R(t)$ and $Q(t)$. Such calculations became possible once ice-core data provided a record of past $\mathrm{CO}_{2}$ concentrations. Enting \& Mansbridge (1987) gave a Laplace transform analysis of the inversion. They obtained an inversion relation which, in the present notation is

$$
\tilde{S}(p)=p \tilde{Q}(p)[1+\tilde{B}(p)]=\tilde{\dot{Q}}(p)[1+\tilde{B}(p)]
$$

where $\tilde{\dot{Q}}(p)$ denotes the Laplace transform of the rate of change of $Q(t)$ with time. This calculation requires numerical differentiation of ice-core data - an inherently ill-conditioned process, but one that is inherent in the inversion. However Enting \& Mansbridge (1987) showed that the need for the derivative could be restricted to the time of the source estimate, using a second inversion relation

$$
\tilde{S}(p)=p \tilde{Q}(p)+\tilde{Q}(p)[p \tilde{B}(p)]=\tilde{\dot{Q}}(p)+\tilde{Q}(p) B(t=0)+\tilde{Q}(p) \tilde{\dot{B}}(p)
$$

In principle, the factor $B(t=0)$ can be obtained from approximants as the $p \rightarrow \infty$ limit of $\tilde{B}(p)$, given by the ratio of the highest order coefficients in numerator and denominator of $[M, N]$ approximants. For approximants based on the expansion about $p=0$ such estimates are likely to be poor.

The deconvolution operation enters implicitly in the study of greenhouse gas metrics by Enting \& Clisby (2021). They considered the problem of finding the $\mathrm{CO}_{2}$-equivalent of methane emissions, $S_{\mathrm{CH} 4}$. They based their analysis on radiative forcing as an equivalent influence on climate (Wigley 1998) which requires

$$
a_{\mathrm{CH} 4} \tilde{R}_{\mathrm{CH} 4}(p) \tilde{S}_{\mathrm{CH} 4}(p)=a_{\mathrm{CO} 2} \tilde{R}_{\mathrm{CO} 2}(p) \tilde{S}_{\text {equiv }}(p)
$$


I. G. Enting, Pade-Laplace in carbon-climate interactions.

with $a_{\mathrm{CO} 2}$ and $a_{\mathrm{CH} 4}$ as the radiative forcing per unit mass. Equivalence is specified by

$$
\tilde{S}_{\text {equiv }}(p)=\frac{a_{\mathrm{CH} 4}}{a_{\mathrm{CO} 2}} \Psi(p) \tilde{S}_{\mathrm{CH} 4}(p) \approx \frac{a_{\mathrm{CH} 4}}{a_{\mathrm{CO} 2}} \frac{\tilde{R}_{\mathrm{CH} 4}(p)}{\tilde{R}_{\mathrm{CO} 2}(p)} \tilde{S}_{\mathrm{CH} 4}(p)=\frac{a_{\mathrm{CH} 4}}{a_{\mathrm{CO} 2}} \frac{p \tilde{R}_{\mathrm{CH} 4}(p)}{p \tilde{R}_{\mathrm{CO} 2}(p)} \tilde{S}_{\mathrm{CH} 4}(p)
$$

where $\Psi$ operates on $S_{\mathrm{CH} 4}$ to generate an index that defines equivalence. Comparing the rightmost expression above to equaton (5) shows that $\Psi(p)$ can be regarded as an approximation to the ratio of airborne fractions of $\mathrm{CH}_{4}$ and $\mathrm{CO}_{2}$. The Global Warming Potential approximates $\Psi$ as a constant. Other approaches use $\Psi$ as a derivative operator (Smith et al. 2012, Lauder et al. 2013, and later work). Enting \& Clisby (2021) analysed these and more recent metrics as Laplace transforms and considered $\Psi(p)$ as a low-order approximation to $\tilde{R}_{\mathrm{CH} 4}(p) / \tilde{R}_{\mathrm{CO} 2}(p)$.

Response functions can also be used in deconvolution calculations either directly in a mass-balance form (Wigley 1991) or as statistical estimation via the Kalman filter (Trudinger et al. 2002).

\section{FEEDBACK}

Studies of global change have increasingly focused on the extent to which the influence of climate change on the carbon cycle can create a feedback loop that amplifies the greenhouse effect. Enting (2010) described the linearised behaviour for warming, $W(t)$, in terms of Laplace transforms, as

$$
\tilde{W}(p)=a_{\mathrm{CO} 2} \tilde{U}(p) \tilde{Q}(p)
$$

with $U(t)$ describing the temperature response to radiative forcing, and

$$
\tilde{Q}(p)=\tilde{R}(p)[\tilde{S}(p)+\tilde{H}(p) \tilde{W}(p)]=\frac{\tilde{S}(p) / p}{1+\tilde{B}_{\mathrm{L}}(p)+\tilde{B}_{\mathrm{O}}(p)}+\frac{\tilde{H}(p) \tilde{W}(p)}{1+\tilde{B}_{\mathrm{L}}(p)+\tilde{B}_{\mathrm{O}}(p)}
$$

leading to relations for the loop gain, $\tilde{G}(p)$ :

$$
\tilde{Q}(p)=\frac{\tilde{R}(p)}{1-a_{\mathrm{CO} 2} \tilde{U}(p) \tilde{H}(p) \tilde{R}(p)} \tilde{S}(p)=\frac{\tilde{R}(p)}{1-\tilde{G}(p)} \tilde{S}_{P}=\tilde{R}_{\mathrm{FB}}(p) \tilde{S}(p)
$$

The feedback also amplifies the warming response, $\tilde{U}(p)$, by a factor $1 /(1-\tilde{G}(p))$ (Enting 2010).

\section{Carbon-climate loop gain}

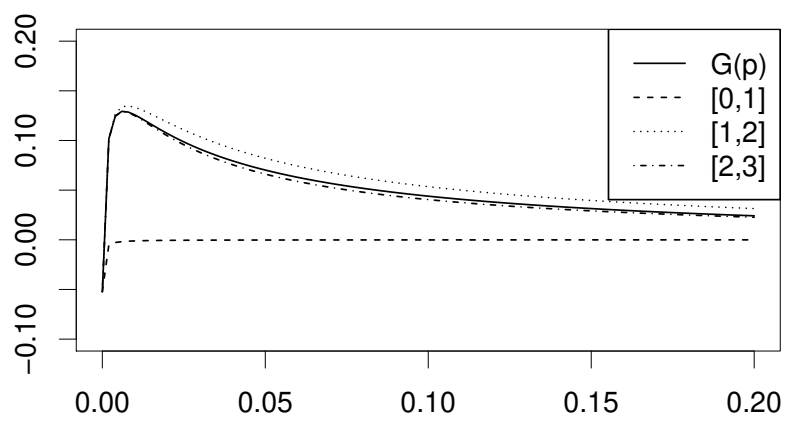

$\mathrm{p}$

Figure 3. Solid line is Laplace transform, $\tilde{G}(p)$, of feedback loop gain, calculated as $1-\tilde{R}_{\text {no feedback }} \tilde{R}_{\text {feedback }}$ using estimates from Joos et al. (2013) (supplementary information). Other lines are Padé approximants. The negative gain at very long timescales is an artifact of the original fits only using 1000 years of model output.

Enting (2011) noted that when response functions, or the models from which they are derived, are calibrated against 20th century data, they will be implicitly including the effects of climate-to-carbon feedback and so will give estimates of $R_{\mathrm{FB}}$ rather than $R$. Joos et al. (2013) gave an example where the feedback had been explicitly excluded. This makes it possible to combine estimates of $\tilde{R}_{\text {no feedback }}$ and $\tilde{R}_{\text {feedback }}$ to give an estimate of the loop gain, $\tilde{G}(p)$. This is shown in Figure 3, along with Padé approximants. The negative gain at very long timescales is an artifact of the original fits only using 1000 years of model output. Nevertheless, the [1,2] and $[2,3]$ approximants can follow the function from an unphysical starting point and give reasonable fits over the more relevant decadal to century timescales. 
I. G. Enting, Pade-Laplace in carbon-climate interactions.

\section{GAMMA}

A widely cited paper, (Friedlingstein et al. 2003), expressed the atmospheric carbon content as

$$
Q(t)=\frac{\int S(t)}{1+\beta_{\mathrm{L}}+\beta_{\mathrm{O}}}+\frac{\left[\gamma_{\mathrm{L}}+\gamma_{\mathrm{O}}\right] \Delta T}{1+\beta_{\mathrm{L}}+\beta_{\mathrm{O}}}
$$

influencing numerous studies that have attempted to estimate $\gamma$ (see citations by Enting \& Clisby 2019). Comparison with (12) reveals that (14) ignores timescale dependence and replaces functions of $p$ by constants. From (12) there is not expected to be a single $\gamma$, applicable on all timescales. and the $\beta$ factors also have a timescale dependence as shown in Figure 2. In addition, if the factor $1 /\left(1+\beta_{\mathrm{L}}+\beta_{\mathrm{O}}\right)$ is replaced by $p \tilde{R}(p)$, the available estimates of the $\mathrm{CO}_{2}$ response are usually estimates of $R_{\mathrm{FB}}$ either explicitly or implicitly if calibrated against 20 th century data.

Rubino et al. (2016) analysed pre-industrial data using a regression analysis based on the time-domain form of (12). They parameterised $H(t)$ as $\theta_{\gamma} H^{*}(t)$ where $H^{*}(t)$ was a unit pulse followed by a relaxation with a 100 -year timescale. The regression fitted $\iint R\left(t-t^{\prime}\right) H^{*}\left(t^{\prime}-t^{\prime \prime}\right) W\left(t^{\prime \prime}\right) d t^{\prime \prime} d t^{\prime}$ to $M(t)$ from ice-core data using various paleo-temperature data sets for $W(t)$. For the 20th century, Enting \& Clisby (2019) added a term $\theta_{s} \int R\left(t-t^{\prime}\right) S\left(t^{\prime}\right) d t^{\prime}$ to be fitted, where $\theta_{s}$ was an ad hoc correction to account for the use of $R_{\mathrm{FB}}$ in place of $R$. The gain estimate shown in Figure 3 should provide a better correction than ad hoc use of a constant.

\section{CONCLUSIONS}

This paper illustrates Padé-Laplace analysis in the study of $\mathrm{CO}_{2}$ and climate. Padé approximants as approximations to Laplace transforms of response functions, and combinations thereof, give expressions which are less subject to ill-conditioning in the specification and give simple closed-form expressions in the time domain. Most of the examples here fit Maclaurin series in the transform variable $p$, making them only applicable to long timescale aspects. Approximants based on Taylor series provide scope for more general analyses.

\section{NOTATION}

Laplace transforms are denoted by the tilde notation, e.g. $\tilde{R}(p)$ as the Laplace transform of $R(t)$.

$a_{\mathrm{X}}$ Radiative forcing per unit mass of constituent $X$.

$B(t)$ Operator specifying the feedback from concentration changes.

$G(t)$ Response function giving gain in carbon-climate feedback loop.

$H(t)$ Function describing $\mathrm{CO}_{2}$ flux as response to temperature change.

$M(t)$ Atmospheric content of carbon (as $\mathrm{CO}_{2}$ ). Perturbation is $Q(t)=\Delta M(t)$.

$p$ Argument of Laplace transform. Equivalent to $e$-folding rate for exponentially growing emissions.

$R_{\mathrm{CH} 4}(t)$ Atmospheric response function for emissions of methane.

$R(t)$ Atmospheric response function for $\mathrm{CO}_{2}$. Various special cases are $R_{\mathrm{FB}}(t), R_{\mathrm{O}}(t)$.

$S(t)$ Anthropogenic emission of $\mathrm{CO}_{2}$.

$t$ Time.

$U(t)$ Response function giving warming as response to radiative forcing.

$W(t)$ Warming from greenhouse effect; also other temperature changes.

$\alpha e$-folding rate of exponentially growing emissions.

$\beta, \beta_{\mathrm{L}}, \beta_{\mathrm{O}}$ Factor specifying feedback from change in $\mathrm{CO}_{2}$ concentration, land and ocean components.

$\gamma, \gamma_{\mathrm{L}}, \gamma_{\mathrm{O}}$ Factor specifying feedback from change in global temperature, land and ocean components.

$\tilde{\Psi}(p)$ Laplace transform of an operator giving an index, derived from $\mathrm{CH}_{4}$ emissions, giving $\mathrm{CO}_{2}$ equivalence.

\section{ACKNOWLEDGEMENT}

The author thanks Nathan Clisby for extensive discussions on this topic. 
I. G. Enting, Pade-Laplace in carbon-climate interactions.

\section{REFERENCES}

Claverie, P., Denis, A. \& Yeramian, E. (1989), 'The representation of functions through the combined use of integral transforms and Padé approximants: Padé-Laplace analysis of functions as sums of exponentials', 9, 247-299.

Enting, I. G. (1990), 'Ambiguities in the calibration of carbon cycle models', Inverse Problems 6, L39-L46.

Enting, I. G. (2007), 'Laplace transform analysis of the carbon cycle', Environmental Modelling and Software 22, 1488-1497.

Enting, I. G. (2010), Inverse problems and complexity in earth system science, in R. L. Dewar \& F. Detering, eds, 'Complex Physical, Biophysical and Econophysical Systems.', World Scientific, Singapore.

Enting, I. G. (2011), 'Seeking carbon-consistency in the climate-science-to-policy interface', Biogeochemistry 104, 59-67, DOI: 10.1007/s10533-099-9351-7, (available on-line 2009).

Enting, I. G. \& Clisby, N. (2019), 'Estimating climatic influence on the carbon cycle', Earth System Dynamics Discussions pp. ESD-2019-41.

Enting, I. G. \& Clisby, N. (2021), 'Technical note: On comparing greenhouse gas emission metrics', Atmospheric Chemistry and Physics 21, 4699-4708.

Enting, I. G. \& Mansbridge, J. V. (1987), 'Inversion relations for the deconvolution of $\mathrm{CO}_{2}$ data from ice cores.', Inverse Problems 3, L63-69.

Enting, I. G., Wigley, T. M. L. \& Heimann, M. (1994), Future emissions and concentrations of carbon dioxide: Key ocean/atmosphere/land analyses, Technical report, CSIRO. at http://www.cmar.csiro.au/eprint/open/enting_2001a0.htm.

Feldman, P. \& Freund, R. W. (1995), 'Efficient linear circuit analysis by Padé approximation via the Lanczos process', IEEE Trans Computer aided design of integrated circuits and systems 14, 639-649.

Friedlingstein, P., Dufresne, J.-L., Cox, P. M. \& Rayner, P. (2003), 'How positive is the feedback between climate change and the carbon cycle?', Tellus 55B, 692-700.

Gregory, J. M., Jones, C. D., Cadule, P. \& Friedlingstein, P. (2009), 'Quantifying carbon cycle feedbacks', J. Climate 22, 5232-5250.

Joos, F., Roth, R., Fuglestvedt, J. S., Peters, G. P., Enting, I. G., von Bloh, W. et al. (2013), 'Carbon dioxide and climate impulse response functions for the computation of greenhouse gas metrics: a multi-model analysis', Atmos. Chem. Phys. 13, 2793-2825.

Lanczos, C. (1956), Applied Analysis, Prentice Hall, Englewood Cliffs, N.J.

Lauder, A. R., Enting, I. G., Carter, J. O., Clisby, N., Cowie, A. L., Henry, B. K. \& Raupach, M. (2013), 'Offsetting methane emissions - an alternative to emission equivalence metrics', International Journal of Greenhouse Gas Control 12, 419-429.

Meinshausen, M., Raper, S. C. B. \& Wigley, T. M. L. (2011), 'Emulating coupled atmosphere-ocean and carbon cycle models with a simpler model: — part 1 model description and calibration MAGICC6', Atmospheric Chemistry and Physics 11, 1417-1456, doi:10.5194/acp-11-1417-2011.

Oeschger, H. \& Heimann, M. (1983), 'Uncertainties of predictions of future atmospheric $\mathrm{CO}_{2}$ concentrations', J. Geophys. Res. 88C, 1258-1262.

Oeschger, H., Siegenthaler, U. \& Heimann, M. (1980), The carbon cycle and its perturbations by man, in W. Bach, J. Pankrath \& J. Williams, eds, 'Interactions of Energy and Climate', Reidel, Dordrecht, pp. 107127.

Rubino, M., Etheridge, D. M., Trudinger, C. M., Allison, C. E., Rayner, P. J., Enting, I., Mulvaney, R., Steele, L. P., Langenfelds, R. L., Sturges, W. T., Curran, M. A. J. \& Smith, A. M. (2016), 'Low atmospheric $\mathrm{CO}_{2}$ levels during the Little Ice Age due to cooling-induced terrestrial uptake', Nature Geosci 9, 691-694 http://dx.doi.org/10.1038/ngeo2769L3.

Smith, S. M., Lowe, J. A., Bowerman, N. H. A., Gohar, L. K., Huntingford, C. \& Allen, M. R. (2012), 'Equivalence of greenhouse-gas emissions for peak temperature limits', Nature Climate Change 2, 535538.

Trudinger, C. M., Enting, I. G., Rayner, P. J. \& Francey, R. J. (2002), 'Kalman filter analysis of ice core data. 2 Double deconvolution of $\mathrm{CO}_{2}$ and $\delta^{13} \mathrm{C}$ measurements', J. Geophys. Res. 107, 4423, doi: 1029/2001JD001112.

Wigley, T. M. L. (1991), 'A simple inverse carbon cycle model', Global Biogeochemical Cycles 5, 373-382.

Wigley, T. M. L. (1998), 'The Kyoto Protocol: $\mathrm{CO}_{2}, \mathrm{CH}_{4}$ and climate implications', Geophys. Res. Lett. 25, 2285-2288.

Yeramian, E. \& Claverie, P. (1987), 'Analysis of multiexponential functions without a hypothesis as to the number of components', Nature 326, 169-174. 